\title{
Aliseda 18. Muralismo desde la participación ciudadana, para la recuperación urbana
}

\author{
MARÍA ANTONIA FERNÁNDEZ NIETO \\ Doctor Arquitecto. Profesora Escuela de Arquitectura de la Universidad Francisco de Vitoria. \\ Coordinadora del Grupo de Investigación Procesos Arquitectónicos y Estrategias Urbanas \\ 12485fernandez@coam.es \\ EDUARDO ZAMARRO FLORES \\ Doctor en Bellas Artes. Profesor Escuela de Arquitectura y Bellas Artes de la Universidad \\ Francisco de Vitoria. Grupo de Investigación Procesos Arquitectónicos y Estrategias Urbanas \\ pintor@eduardozamarro.com
}

\begin{abstract}
Resumen
Se presenta un caso práctico donde la participación ciudadana actúa en la transformación de espacios degradados de la ciudad. Aliseda18 es un huerto urbano de la primera periferia de Madrid. Dentro de esta iniciativa se plantea una pintura mural como elemento vertebrador y unificador del nuevo espacio. Como el resto de actuaciones, este elemento se realiza con la participación de distintos colectivos de distintas edades que trabajan de forma transversal en la creación del mural desde sus planteamientos e ideas iniciales hasta la propia realización. Lo que eran unas medianeras sin identidad se transforman en un telón de fondo que da identidad al huerto urbano, tanto para sus usuarios como para el resto del barrio. Desde la escala local se pueden producir procesos de reapropiación del espacio público y de generación de paisaje urbano que responden a iniciativas de sus habitantes. Mediante la participación en las decisiones sobre el entorno y en su misma configuración, los ciudadanos dotan de identidad a la ciudad que habitan, expresan su diversidad social y cultural, y se sienten identificados y responsabilizados con ella.
\end{abstract}


Desde un proyecto de investigación de la Universidad Francisco de Vitoria se trabaja de forma transversal realizando sucesivos avances en la recopilación de información, la relación con otras experiencias y la aplicación en un caso concreto. De esta manera la investigación y creación académica se implica con la transformación de la realidad y se enriquece al ponerse en práctica.

Palabras clave: Muralismo; participación ciudadana; recuperación urbana; periferia.

\title{
Aliseda 18. Mural from citizen participation, for urban recovery
}

\begin{abstract}
We present a study case where citizen participation acts in transforming degraded areas of the city. Aliseda18 is an urban garden in the first outskirts of Madrid. Within this initiative arises a mural as unifying backbone of the new space. Like all other performances, this item is made with the participation of different groups of different ages who work in the creation of the mural from their initial proposals and ideas to actual implementation. What were some medians without identity are transformed into a backdrop that gives identity to the urban garden, both for users and for the rest of the neighborhood.

From the local level can produce processes of reappropriation of public space and urban landscape generation initiatives that respond to their inhabitants. Through participation in decisions on the environment and in the same configuration, citizens equip identity to the city they inhabit, express their social and cultural diversity, and are identified and held accountable to it.

From a research project at the University Francisco de Vitoria lot of work is being made in a cross made successive advances in information gathering, relationship with other experiences and application in a particular case. Thus research and academic development involves the transformation of reality and enriches by implementing.
\end{abstract}

Key words: Mural; citizen participation; urban recovery; suburbs.

Sumario: 1.- Introducción. 2.- Antecedentes. 3.- Análisis del caso. 4.- Conclusión. 5.Referencias bibliográficas. 


\section{Introducción.}

El trabajo mural nació como una intervención más en la recuperación de un espacio degradado del barrio de Puerta Bonita de Carabanchel planteado desde la asociación del barrio Paso a Paso en Pan Bendito ${ }^{1}$ y haciendo partícipes a los vecinos y asociaciones del lugar. El fin más específico de la intervención era la creación de un huerto urbano comunitario para el barrio.

Dentro del marco investigador del grupo "[AAOO*] ARQUITECTURAS OCASIONALES" de la Escuela de arquitectura de la Universidad Francisco de Vitoria de Madrid $^{2}$ esta propuesta ciudadana se planteó como una oportunidad para desarrollar una vía de investigación práctica en el entendimiento de un nuevo urbanismo y forma de construir la ciudad desde y para la ciudadanía. Esta oportunidad desembocó en el Proyecto de investigación Aliseda 18. Espacio participativo de recuperación urbana.

Todas las tardes durante una semana los vecinos, colegios, asociaciones y todos los agentes socioculturales de la zona de Carabanchel estaban invitados a participar en un "taller de muralismo" que se integraba en otros talleres de

\footnotetext{
${ }^{1}$ La asociación Paso a Paso en Pan Bendito (pasoapasopanbendito@gmail.com) tiene como fines promover, desarrollar y financiar acciones de cooperación para el desarrollo en nuestro entorno en las comunidades sociales más desfavorecidas: inmigrantes, mujeres, parados y paradas de larga duración, minorías étnica y en personas en riesgo de exclusión social. Y favorecer una formación integral de las personas fomentando valores de solidaridad, justicia, democráticos y reciprocidad. Estos fines se desarrollan a través de varias actividades como Banco de Tiempo, Taller de Emprendedores, huerto urbano Aliseda18...

${ }^{2}$ El grupo de investigación Grupo de Investigación sobre Procesos Arquitectónicos y Estrategias Urbanas [AAOO*] ARQUITECTURAS OCASIONALES propone un registro de actividad muy amplio, planteando la superposición de una visión global a propuestas de intervención local. No se trabaja sabiendo cómo van a ser los proyectos, sino que se trabaja a partir de estructuras que permiten desarrollar estrategias inesperadas. De este modo, se abren líneas de trabajo que intercalan objetivos, manifestando las divergencias entre la ciudad planificada y los desarrollos espontáneos, permitiendo hacer visible la complejidad de la ciudad contemporánea, y desarrollando sistemas constructivos y de gestión alternativos. SURGE con vocación de identificar, activar y gestionar nuevos mecanismos proyectivos, desmarcándose de sistemas establecidos. Se interpreta la ciudad como dispositivo redistribuidor de lo valioso, analizando los diferentes sistemas de reapropiación y narración del espacio urbano contemporáneo.
} 
autoconstrucción. La ejecución de este trabajo de pintura mural ha sido un elemento más de huerto urbano, ha unido a la gente en su realización y ha convertido el espacio es un lugar con una nueva identidad colectiva. Niños de colegios y entidades sociales, jóvenes, ancianos, familias enteras y alumnos universitarios ${ }^{3}$, ha sido los ejecutores de este trabajo bajo la dirección del Profesor y artista madrileño Eduardo Zamarro ${ }^{4}$.

\section{Antecedentes.}

En momentos de crisis a lo largo de la historia se han habilitado espacios de la ciudad para la producción hortícola. En situaciones de desabastecimiento de las ciudades los huertos urbanos se convirtieron en una necesidad de autoconsumo. Tanto en la primera como en la segunda guerra mundial aparecen en toda Europa y en Estados Unidos. También se utilizaron como una ayuda a los bajos salarios de los obreros en la revolución industrial del siglo XIX. En la década de los setenta con la crisis energética los huertos urbanos surgieron como ámbito de relación y apoyo comunitario (Morán, 2010).

Sin embargo en la actualidad la creación de huertos urbanos también responde a una necesidad de recuperar la naturaleza y sus ciclos en la vida diaria de los ciudadanos. Desde disciplinas como el ecologismo se intenta reactivar la relación entre campo-ciudad.

Dentro de este ámbito han surgido iniciativas en España y en concreto en Madrid, que desde distintas posiciones entienden los huertos urbanos no solo como una labor hortofrutícola sino como espacios de relación vecinal y modelos de vivir la ciudad alternativos a la sociedad de consumo. Además se han generado redes entre ellos que actúan como interlocutores con los entes

\footnotetext{
${ }^{3}$ Participaron en el proyecto: Asociación Pan Bendito desde su trabajo de ludoteca y apoyo escolar, Taller de creación urbana de Comillas, Colegio Concertado Santa María Micaela, Asociación Ymca, Universidad Francisco de Vitoria

${ }^{4}$ www.eduardozamarro.com
} 
de gobierno de las ciudades y que suponen una dinamización de actividades para la ciudad 5 .

Los huertos también desarrollan un espacio de creación muy importante en las ciudades ofreciendo oportunidades a artistas para acondicionar los espacios o bien como el caso de Aliseda18 realizando intervenciones colectivas.

El caso del huerto urbano Aliseda18 se encuentra integrado dentro de estas iniciativas madrileñas y se encuadra en una primera periferia de los años 50-60 con una estructura urbana de bloque abierto. Este modelo morfológico de ciudad generó en su día espacios "interbloques" que en estos momentos son vacíos urbanos que se pueden entender como espacios degradados, pero también como espacios de oportunidad.

\section{Análisis del caso.}

Para un trabajo de este tipo no solo es importante acertar con la idea y el boceto, es tan importante que las técnicas y el desarrollo de las jornadas sean coherentes y permitan la participación de un grupo muy dispar tanto en número como en formación y edad.

En la realización de este trabajo los alumnos de la UFV realizaron la tarea de asistir a los grupos y de ir hilando las intervenciones diarias para llegar a un resultado final que estaba establecido en un plan escrito como una ruta en un boceto, con un fin claro, pero con varias rutas. De esta forma aunado técnicas y el calendario de grupos que participarían cada tarde, los alumnos de la UFV dirigidos por Eduardo Zamarro, preparaban los materiales y los objetivos que se tenían que cumplir cada jornada, básicamente estos fueron los pasos:

5 La Red de Huertos Urbanos comunitarios de Madrid REHD MAD! (http://redhuertosurbanosmadrid.wordpress.com) es una red de redes, para que los colectivos que se dediquen a la agricultura urbana de forma ecológica, que sean públicos y comunitarios, tengan un espacio de encuentro y comunicación. Los huertos Urbanos que forman parte de la red son: Ciudades Comestibles, Esta es una Plaza, Fravm, Grama, Huerta de Cantarranas, Solar de Antonio Grillo, Kybele, Sabia Bruta, El Huerto del Retiro, El Tablao de la Compostura, Ies Barajas, Tabacalera, Revuelta en la Huerta, El Huerto Ambulante y Huerto Aliseda. 
Sanear la superficie con una mano de pintura.

Hacer un primer fondo de hojas, siguiendo unas líneas marcadas en la pared, se prepararon mezclas de colores y tampones con esponjas para hacer forma de hojas de aliso, aquí participaron niños de 5 años en adelante.

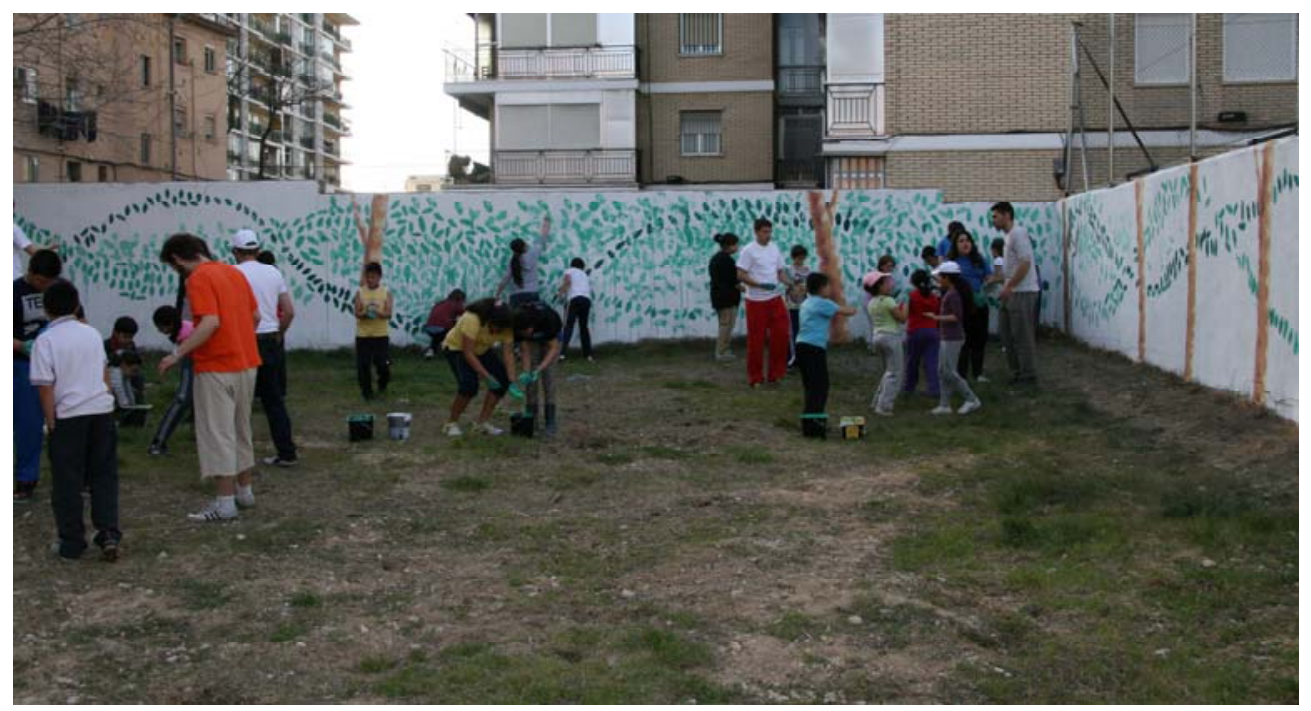

Fig. 1. Trabajo de estampación de hojas con niños.

Taller de siluetas, los participantes en taller muralístico estaban invitados a dejar su silueta en el muro del huerto, para esta tarea necesitábamos a gente de 14 años en adelante, que pudiera calcar la sombra de una persona en un papel con un foco y un carboncillo, luego realizamos estarcidos perforando la silueta que serían replantadas cuando el fondo estuviera terminado, este taller duró varias tardes para representar en el mural los distintos tipos de personas que participan en el huerto.

Sobre el fondo de hojas se realizaron los trocos de los alisos con rodillos, jugando con diferentes espesores de pintura y texturas, estos troncos se hicieron en serie con varias manos, lo que garantizaba una alta participación y que todos los trabajos realizados quedaran integrados en un mismo lenguaje plástico. 
Sobre estos troncos fueron creciendo más hojas, hasta crear un fondo con más densidad al final de huerto y menor en el principio, se volvieron a utilizar los tampones y todas las personas estaban invitadas a participar.

Una vez terminados los fondos, se realiza con adolescentes y adultos el replanteo de las siluetas, los textos que se manuscribieron con barras de óleo y terminamos el trabajo con una plantilla para poner el nombre del huerto, ALISEDA 18 en la tapia final del muro, la que más visibilidad tiene desde la calle de acceso.

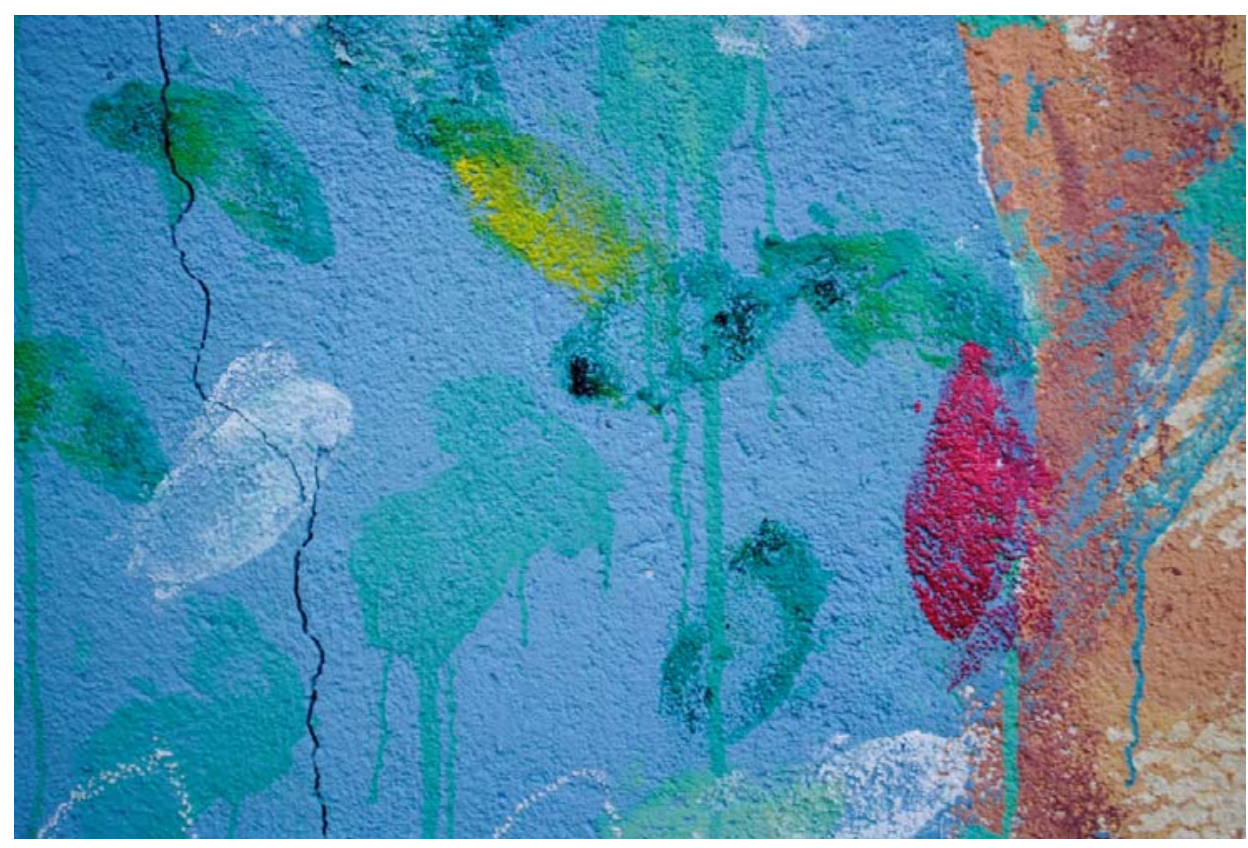

Fig. 2. Detalle de texturas. 


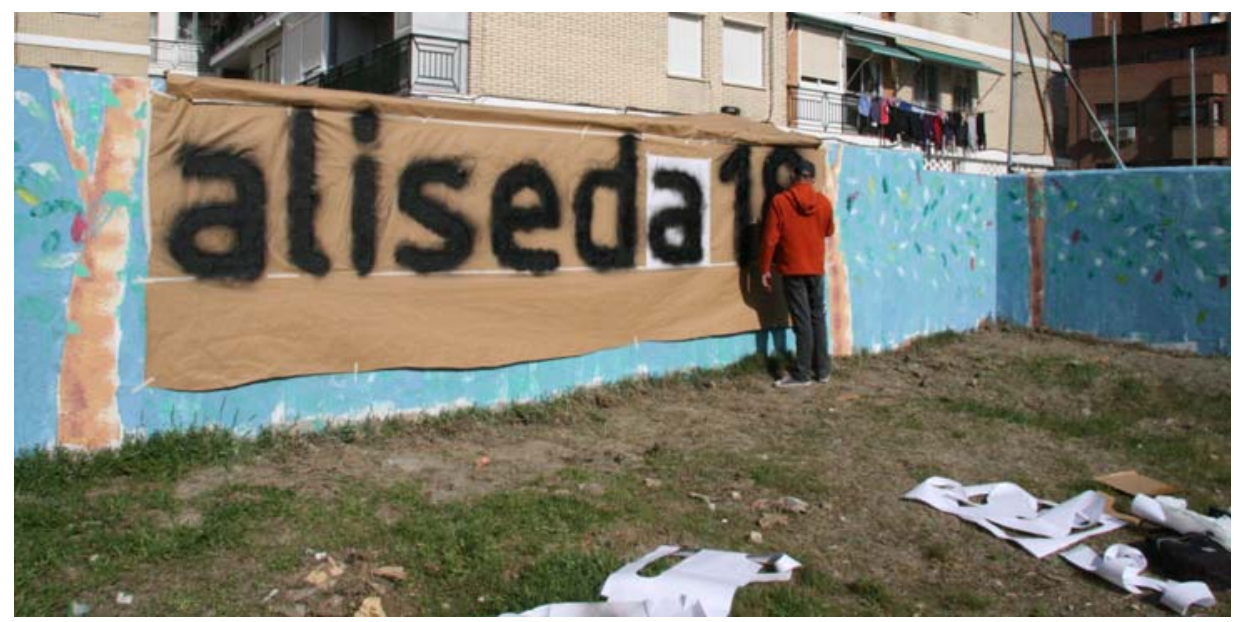

Fig. 3. Ejecución de nombre del huerto.

La pintura utilizada en todos los procesos fue pintura plástica y sus versiones más artísticas en emulsiones acrílicas todos con base agua, los pigmentos utilizados estaban previamente hidratados y no tenían componentes tóxicos ni para las personas ni para la futura actividad hortofrutícola.

Todos los pinceles y rodillos se limpiaban con base agua y fueron reutilizados todos los días, mucho de este material provenía de murales anteriores y los materiales que no se desgastaron con el uso siguen siendo útiles, entendiendo que la mejor manera de reciclar es reutilizar.

El material para la realización de las plantillas y los estarcidos fue papel reciclado. Para este tipo de actividades siempre es importante que los materiales no sean peligrosos, dado el gran margen de edades que trabajaban juntas y la poca o nula experiencia en actividades artísticas. En estos casos el arte funciona como un catalizador de fuerzas individuales hacia la realización de un fin común. 


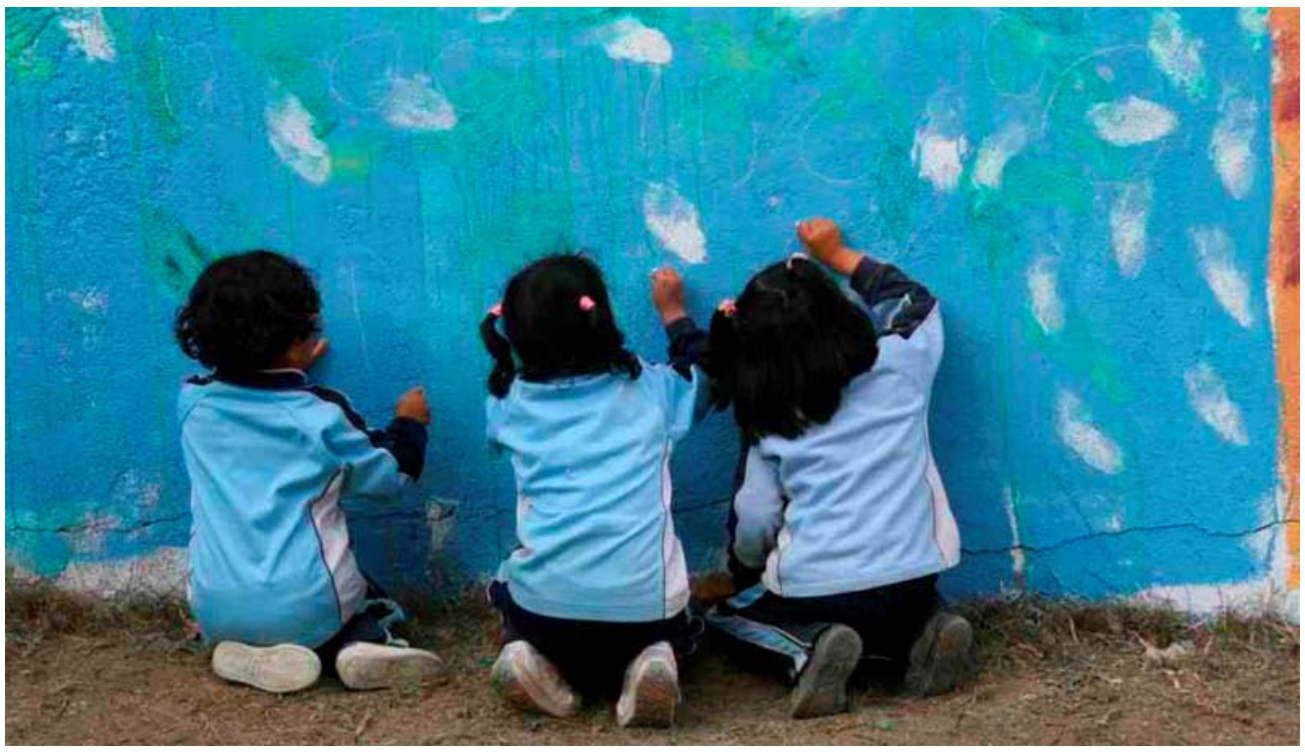

Fig. 4. Niños participando.

Lo más importante para garantizar una buena intervención es que las técnicas y los equipos no interfieran en el desarrollo del mural comunitario, que cada día crezca la idea inicial gracias a la aportación de todos, eso permite que estas intervenciones no se aprecien como imposiciones externas sino como obras de una autoría colectiva, aunque nunca hay que olvidar que sin una dirección eficaz, tanto en lo artístico como en lo técnico estas intervenciones difícilmente terminarían en la consecución de un plan ordenado y coherente. Lo más importante es implicar al colectivo con una propuesta interesante, que recoja sus inquietudes e ideas, si no hay gente participando no hay mural colectivo, no hay relación entre arte y sociedad.

\section{Conclusión.}

En la actualidad se están produciendo acciones ciudadanas de apropiación del espacio público obsoleto, degradado o en desuso que suponen un nuevo entendimiento de la forma de hacer ciudad. Esta manera de hacer ciudad, de abajo a arriba, plantea cambios sociológicos y urbanísticos que afectan tanto a las relaciones ciudadanas como a la forma de vivir la ciudad. 
Dentro de estos espacios, en unas ciudades con periferias monótonas, la expresión artística es parte importante de la generación de identidad para sus habitantes. La participación de los mismos en las obras hace que se identifiquen, se responsabilicen y se apropien de ellas.

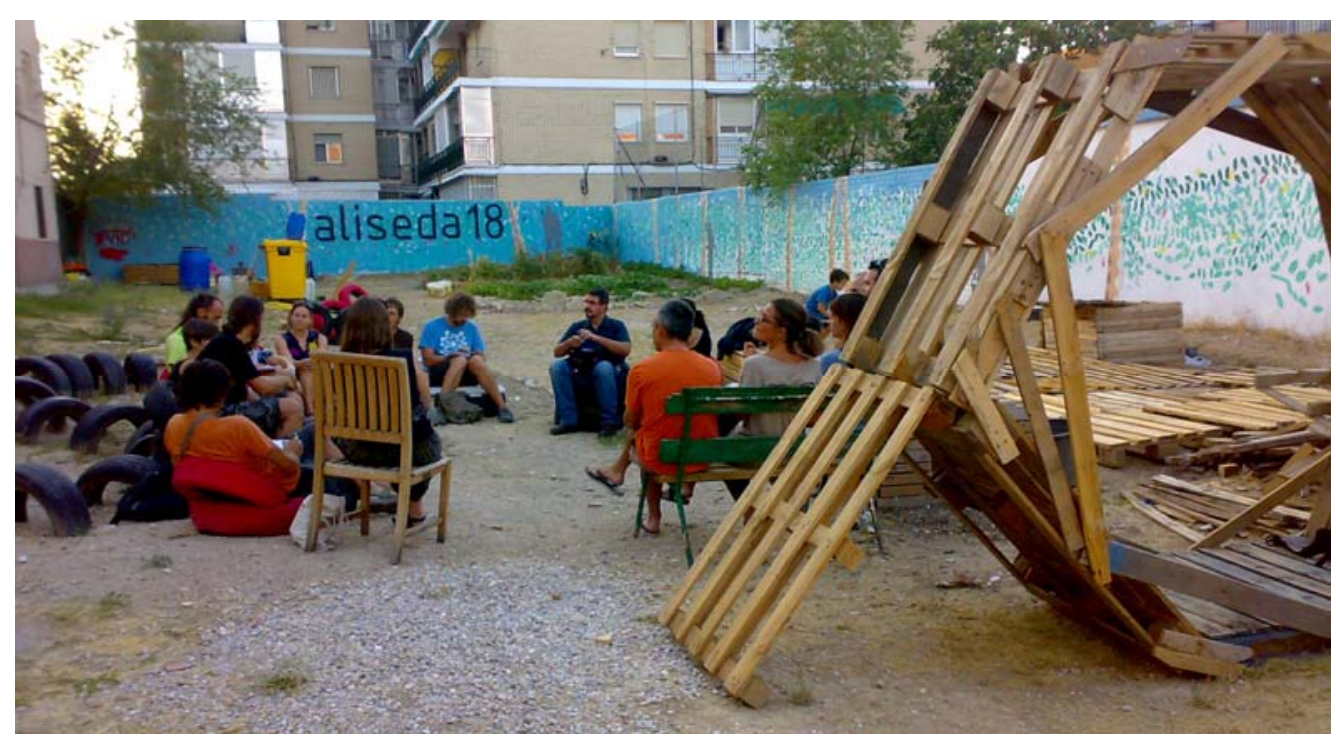

Fig. 5. Reunión de Red de Huertos Urbanos en Aliseda 18.

La oportunidad de que artistas y colectivos participen en un proyecto único cambia los roles habituales entre cliente y artista, haciendo que el proceso sea una parte tan importante como el resultado de la obra.

En el barrio de Puerta Bonita el huerto Aliseda18 ha recuperado un espacio no regulado y abandonado reinventándose en un huerto urbano. Asociaciones de vecinales, colegios, institutos y los propios vecinos se han convertido en los constructores y beneficiarios de este espacio de la ciudad dotándole de identidad propia. 


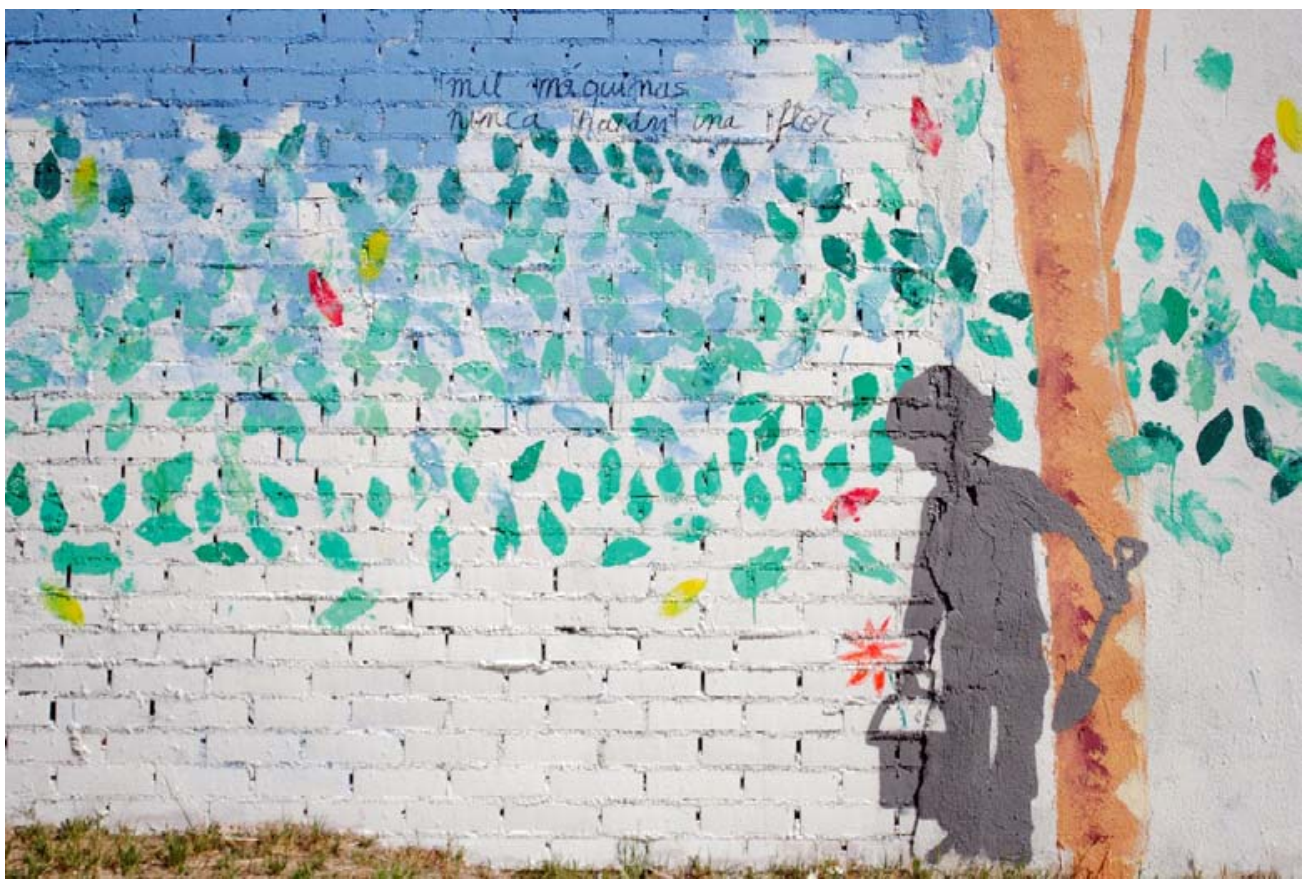

Fig. 6. Mil máquinas nunca harán una flor.

\section{Bibliografía.}

HERNÁNDEZ GARCÍA, Jaime (2008): Arquitectura, participación y hábitat popular. Pontificia Universidad Javeriana, Bogotá.

Hough, Michael (1998): Naturaleza y ciudad. Planificación urbana y procesos ecológicos. Barcelona, Gustavo Gili.

MORÁn AlONSO, Nerea (2008): Huertos y jardines comunitarios. Boletín $C F+S$, 40, págs. 75-124. Disponible en habitat.aq.upm.es/boletin/n40/anmor.html

MORÁN, Nerea (2010): “Agricultura urbana: un aporte a la rehabilitación integral". Papeles de relaciones ecosociales y cambio global.

MOZAS, Javier, (2011): "El espacio público como campo de Batalla". Strategy and Tactics in public space., $a+t$. 